\title{
Minimum mean brightness error bi-histogram equalization in contrast enhancement
}

\begin{abstract}
Histogram equalization (HE) is widely used for contrast enhancement. However, it tends to change the brightness of an image and hence, not suitable for consumer electronic products, where preserving the original brightness is essential to avoid annoying artifacts. Bi-histogram equalization (BBHE) has been proposed and analyzed mathematically that it can preserve the original brightness to a certain extends. However, there are still cases that are not handled well by BBHE, as they require higher degree of preservation. This paper proposes a novel extension of $\mathrm{BBHE}$ referred to as minimum mean brightness error bi-histogram equalization (MMBEBHE) to provide maximum brightness preservation. BBHE separates the input image's histogram into two based on input mean before equalizing them independently. This paper proposes to perform the separation based on the threshold level, which would yield minimum absolute mean brightness error (AMBE - the absolute difference between input and output mean). An efficient recursive integer-based computation for AMBE has been formulated to facilitate real time implementation. Simulation results using sample image which represent images with very low, very high and medium mean brightness show that the cases which are not handled well by $\mathrm{HE}, \mathrm{BBHE}$ and dualistic sub image histogram equalization (DSIHE), can be properly enhanced by MMBEBHE. Besides, MMBEBHE also demonstrate comparable performance with BBHE and DSIHE when come to use the sample images show in [Yeong-Taeg Kim, February 1997] and [Yu Wan et al., October 5 1999].
\end{abstract}

Keyword: Bi-histogram equalization; Dualistic sub-image; Histogram equalization; Minimum mean brightness error 